
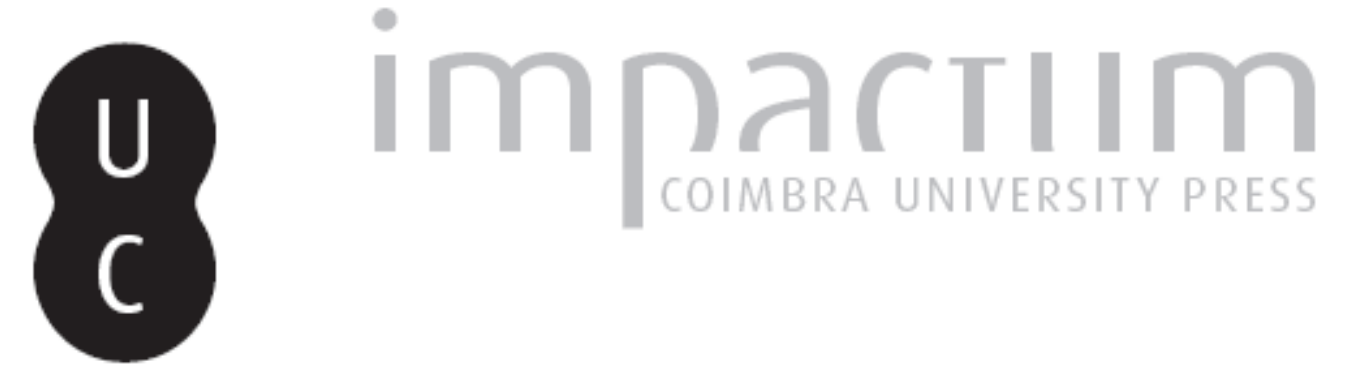

\title{
Los teonimos indigenas com la mención "Deus-A" en la epigrafia hispana
}

Autor(es): Salinas de Frías, Manuel

Publicado por: Imprensa da Universidade de Coimbra

URL persistente:

URl:http://hdl.handle.net/10316.2/45462

DOI:

DOI:https://dx.doi.org/10.14195/1647-8657_34_4

Accessed : $\quad$ 26-Apr-2023 05:54:16

A navegação consulta e descarregamento dos títulos inseridos nas Bibliotecas Digitais UC Digitalis, UC Pombalina e UC Impactum, pressupõem a aceitação plena e sem reservas dos Termos e Condições de Uso destas Bibliotecas Digitais, disponíveis em https://digitalis.uc.pt/pt-pt/termos.

Conforme exposto nos referidos Termos e Condições de Uso, o descarregamento de títulos de acesso restrito requer uma licença válida de autorização devendo o utilizador aceder ao(s) documento(s) a partir de um endereço de IP da instituição detentora da supramencionada licença.

Ao utilizador é apenas permitido o descarregamento para uso pessoal, pelo que o emprego do(s) título(s) descarregado(s) para outro fim, designadamente comercial, carece de autorização do respetivo autor ou editor da obra.

Na medida em que todas as obras da UC Digitalis se encontram protegidas pelo Código do Direito de Autor e Direitos Conexos e demais legislação aplicável, toda a cópia, parcial ou total, deste documento, nos casos em que é legalmente admitida, deverá conter ou fazer-se acompanhar por este aviso.

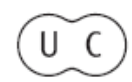


UNIVERSIDADE DE COIMBRA

FACULDADE DE LETRAS

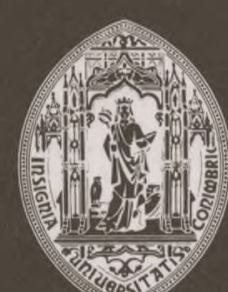

CONIMBRIGA

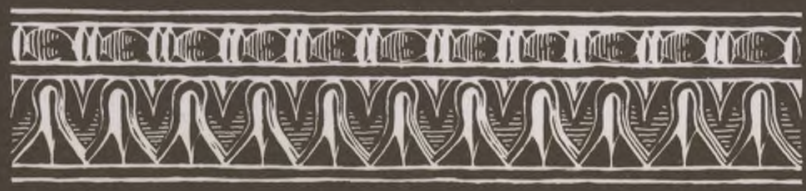

VOLUME XXXIV - 1995

INSTITUTO DE ARQUEOLOGIA 
MANUEl SALINAS DE FRÍAS

Professor da Universidade de Salamanca

LOS TEONIMOS INDIGENAS CON LA MENCION "DEUS, -A"

EN LA EPIGRAFIA HISPANA

"Conimbriga" XXXIV (1995) p. 129-146

RESUmO: Conjunto de observações sobre a temática em epígrafe, com a esperança de contribuir para uma compreensão melhor das realidades religiosas na Hispânia Romana e da sua tradução epigráfica.

Conclui-se que a menção deus, dea, não implica necessariamente a persistência dum culto indígena.

RÉSUMÉ: Un ensemble d'observations dont l'objectif est donner une contribution à une meilleure compréhension des réalités ré ligieuses dans $\boldsymbol{Y}$ Hispania Romana et leur traduction épigraphique.

On conclut que la mention deus, dea, n'est pas un signe évident de la persistance d'un culte indigène. 
(Página deixada propositadamente em branco) 


\section{LOS TEONIMOS INDIGENAS CON LA MENCION "DEUS, -A" EN LA EPIGRAFIA HISPANA $\left(^{(}\right)$}

La intención original de estas líneas había sido la de realizar un estudio sobre las inscripciones hispanas que mencionan los términos deus y dea y el problema de la denominada interpretatio indígena. El desarrollo de nuestra propia investigación, sin embargo, reveló que éste era un objetivo demasiado ambicioso. Lo que sigue, por consiguiente, es una serie de observaciones sobre el conjunto de las inscripciones hispanas en que los teónimos, y en particular los teónimos indígenas, van acompañados de los términos deus y dea. Este conjunto ha dado origen a una teoría, inicialmente expuesta por D. Vaglieri( $\left.{ }^{2}\right)$ pero que fue Lambrino quien popularizó extraordinariamente entre los investigadores hispanos, según la cual lo que subyace bajo dichos teónimos es la existencia de un culto y una divinidad indígena que han sido asimilados, muy frecuentemente, a otros romanos. La mera mención de los términos deus y dea en un epígrafe se convierte así en un indicio o traza de indigenismo religioso en las inscripciones. Esta idea ha sido manejada a menudo indiscriminadamente, de una manera que a nuestro entender contribuye poco a clarificar el conocimiento de las religiones antiguas de la Península Ibérica. Es por ello por lo que queremos presentar el conjunto de observaciones siguientes, con la esperanza de contribuir a una mejor comprensión de las realidades religiosas en la

(') Este artículo se redactó inicialmente como una comunicación al VI Coloquio sobre Linguas e Culturas Paleo-hispánicas. Causas ajenas a nuestra voluntad nos impidieron participar en él. Agradecemos ahora la acogida que la revista Conimbriga le otorga en sus páginas.

(2) S. v. "deus", en el Dizionario epigrafico di Antichità romana, dirigido por E. de RUGGIERO, II,2, 1910. 
Hispania romana y de su traducción epigráfica. Especialmente, hemos intentado una comparación entre lo que conocemos en el ámbito romano y lo que conocemos en el ámbito indígena, puesto que de dicha comparación puedan tal vez deducirse los márgenes más seguros del conocimiento de dichas religiones.

1. Como hemos dicho, dentro de la teonimia peninsular hispánica existe un conjunto particular formado por aquellos casos en que los nombres de los dioses van acompañados de los términos deus, en masculino, o dea, en femenino. Quien primero reparó en esta clase de menciones como algo con una característica peculiar fue Scarlat Lambrino. Como es conocido de todos, al estudiar los cultos indígenas Lambrino distinguió tres etapas en su proceso de romanización. Una primera etapa contemplaba la yuxtaposición e identificación de una divinidad indígena y otra romana entre las cuales los adoradores creían percibir puntos de semejanza; por ejemplo, Cosus Mars. Una segunda etapa contemplaba la substitución del teónimo indígena por la palabra deus o dea (por ej. deus Mars) que era, según él, inútil para un dios romano y que por consiguiente delataba a la antigua divinidad indígena suplantada por él. Y finalmente la tercera etapa consistía en la substitución total del dios indígena por el romano, ahora ya sin la mención de deus, en la misma región o localidad ( ${ }^{3}$ ). La base de este fenómeno vendría dada por la práctica de la interpretatio tanto por parte de indígenas como de romanos.

La teoría de Lambrino hizo fortuna tan rápidamente que la suposición de que en cualquier caso la mención de deus o dea en las inscripciones hispanas enmascara una divinidad indígena ha sido asumida por de casi todos los investigadores, quienes la han aceptado sin criticar ni sus supuestos metodológicos ni los casos concretos propuestos por Lambrino.

Más recientemente, sin embargo, se han propuesto otros enfoques que suponen una cierta rectificación de la opinión general. En el III Coloquio sobre lenguas y culturas paleohispánicas J. Untermann presentó una lista de casos en que los teónimos indígenas aparecían acompañados de un apelativo latino del tipo deus, dominus, Lar, genius o, menos frecuentemente, Iupiter, Mars u otra divinidad romana. La conclusión a que llegaba Untermann era que en el área de su estudio estos términos llenaban un campo semántico que en la lengua indígena

(3) ScARlat LAMBrino, "Les cultes indigènes sous Trajan et Hadrien", Les empereurs romains d'Espagne, Paris 1975, 232. 
llenaban términos como Bandi-, Bandu-, Cosu, Nabla, Reue sin que existiera, sin embargo, una correspondencia exacta entre una lengua y otra $\left({ }^{4}\right)$. Más recientemente, J. d'Encarnação ha demostrado que la sola mención de los términos deus, - $a$ en las inscripciones no significa por sí misma que nos hallemos en presencia de una divinidad indígena, ya que dicha mención puede acompañar tanto a divinidades indígenas, romanas u orientales, como referirse a aquellas que, por estar depositado el epígrafe en su santuario, no aparecen explícitamente en la inscripción (5).

En la hipótesis de Lambrino, y esto es lo importante, si en la Península Ibérica encontramos deus Mars, deus lupiter, deus Mercurius es porque, y sólo porque, el término deus encubre la presencia de una divinidad indígena. Ello sería aceptable si, y sólo si, la evolución del teónimo indígena al teónimo puramente romano se diera in situ, es decir en el mismo lugar o en un entorno geográfico reducido. Pero, para seguir con uno de los ejemplos propuestos por el mismo Lambrino, las inscripciones que relacionan Mars con Cosus proceden de Braga (C/L II, 2418: Cososus Deus Mars), de Santiago de Compostela (C/L II, 5071: Cosus Mars) y de Mongón, cerca del cabo de La Nao en la costa Levantina (C/L II, 5960: Mars Semnus Cosus), es decir muy lejos de los dos anteriores emplazamientos, si bien es cierto que se trata de una dedicatoria realizada por un miembro de una vexillatio de la Legio VII Gemina. De la consideración de los tres epígrafes, y suponiendo que la dedicatoria de Mongón está hecha por un militar desplazado hasta allí pero que porta consigo las creencias y los valores adquiridos en la tierra de su unidad de origen, lo que podemos deducir es que en Galaecia se daba una identificación entre el dios indígena Cosus y el Marte romano, pero no que el culto de Marte o su personalidad terminaran suplantando la de Cosus. Sencillamente, no tenemos ninguna base para afirmarlo.

2. La hipótesis de Lambrino se basa en dos supuestos. El primero de ellos es que el término deus es inútil para un dios romano. Para ser

$\left(^{4}\right)$ J. UntermanN, "LOS teónimos de la región lusitano-gallega como fuente de las lenguas indígenas" Actas del III coloquio sobre lenguas y culturas paleohispánicas, (Lisboa 1980), Salamanca 1985, 358.

${ }^{(5)}$ J. EnCARnaÇão, "Omissão dos teónimos em inscrições votivas" Actas del IV coloquio sobre lenguas y culturas paleohispánicas, Veleia 2-3, 1987, 303-310; "Interpretatio romana. Matériaux pour l'étude de l'acculturation religieuse en Lusitanie" Lengua y cultura en la Hispania prerromana. Actas del $V$ coloquio sobre lenguas $y$ culturas prerromanas de la Península Ibérica, Salamanca 1993, pp. 281-287. 
cierta esta suposición haría falta que los propios romanos no aplicasen nunca esta palabra a sus propios dioses. La más breve consideración muestra que esta suposición es falsa. Tenemos en primer lugar el hecho de que algunas divinidades específicamente romanas sólo eran mencionadas en compañía del apelativo dii, como es el caso de los Di Manes, Di Penates y, más esporádicamente, Di Lares. Tenemos también el caso de la Dea Dia, mencionada en las letanías del colegio de los Arvales, y ya dentro del ámbito epigráfico, un largo elenco de inscripciones en que divinidades genuinamente romanas, como Saturnus, Apollo, Mercurius, Mars, Hercules, Fortuna, Ops, Tellus, o luno, aparecen acompañadas por los términos deus y dea $\left(^{6}\right)$. En un documento muy antiguo, como es la devotio de los Decios, se enumeran Jano, Júpiter, Marte, Quirino, Belona, Lares, Dioses Novensiles, Dioses Indigetes, y los dioses bajo cuya potestad estaban los romanos y sus enemigos (Liv. 8,9,9).

Este uso de los términos deus y dea de daba también en la lengua literaria $\mathrm{y}$, hemos de suponer, en el lenguaje cotidiano también. De los muchos ejemplos recogidos en el Thesaurus linguae latinae $\left({ }^{7}\right)$ señalaremos algunos de Varrón por ser erudito aficionado a las cosas religiosas. Así, en de ling.lat. 5,46 dice: is deus (Vortummus) Etruriae princeps; en 6,21 : Volturnalia a deo Volturno; y en 1,36: Fauni dei Latinorum, ita ut et Faunus et Fauna. Otros ejemplos de este uso son también Plauto, Bacch. 255: Volcanus, Luna, Sol, Dies di quattuor, y Stich. 393: Hercules qui deus sis; Propercio, 4,2,34: Faunus sum deus $\backslash$ Cicerón, Verr. 2, 49: tenes apud Tenedios... sanctissimus deus, nat. deor. 3,43: si Nymphae deae sunt:; Valerio Sorano, Carm. frg. 4: Iuppiter omnipotens rerum regumque repertor (...) deus unus et idem; y Virgilio, Aen. 4,510: deos Erebumque Chaosque tergeminamque Hecatem.

Dentro de la Península Ibérica, no parece tampoco que la mención deus encubra una divinidad indígena cuando encontramos en Valencia ('CIL II, 3726) una inscripción dedicada a Asclepio deo por L. Cornelius Hyginus, sevir augustal cuyo cognomen nos remite a un origen oriental y cuyo status y contexto geográfico y social no muestra la menor traza

(6) Cf., por ejemplo, CIL V, 4854: di sancti Hercules et Iunones; CIL VI, 23.37: deo sancto Apollini pacifero, 467: deus sanctus numen deus magnus Liber Pater, 480: deus sanctus Mars, 30978: deus Mercurius, 531: virgo victrix sancta dea Nemesis, 2826: deus Saturnus patrius, 769: dea Tellus, 771: dea sanctissima Terra mater, 537: dea Nortia; CIL IX, 684: dea Ops; CIL XI,2685: dea Nortia magna.

(7) Thesaurus linguae latinae, Munich 1900, sv. "deus". 
de indigenismo. Ni tampoco es el caso de una inscripción de Velilla, Zaragoza ( $C I L$ 11,3849) en que Lucio y Cornelia, conquiescentes, consagran un predio, probablemente el mismo en el que se erigió su sepultura, sacre Dive Diane. En el caso de la dea o Diva Fortuna (CIL 11,3026, Alcalá H.) estamos frente a una pura expresión clásica; y en las dedicatoria a Isis dea (CIL 11,3387) y deus invictus Mithras (CIL 11,5366 Italica, dedicada por un tal Secundinas) no haya nada que remita o pueda hacer pensar en una divinidad indígena identificada con Mitra o Isis. Por el contrario, estas dedicatorias muestran que la denominación deus o dea puede acompañar indistintamente a teónimos indígenas, grecorromanos $\mathrm{u}$ orientales sin que ello indique que en cada caso bajo dicha denominación se encubre una divinidad autóctona. $\mathrm{O}$ ¿qué pensar entonces de la mención de los dei deaeque en la Lex Urson. 11,1,8?. Fijémonos en una inscripción dedicada a deus Mars (CIL 11,1472) por Septimenus r(ei) p(ublicae) A(stigitanae servus), es decir por un servus publicus de la muy romanizada capital del conventus Astigitanus, perteneciente a la Bética. No hay nada en la inscripción que nos permita relacionar la dedicatoria con una supuesta divinidad indígena sino, totalmente al contrario, con el culto muy extendido y bien atestiguado en esta provincia del Marte romano ${ }^{8}$ ). Lo mismo sucede con otra inscripción dedicada Marti domino por la respublica Murg(itanorum) (ILER, 252). Dos inscripciones dedicadas a Silvano, divinidad itálica, son también reveladoras a este respecto. Una \{ILER 310, Porcuna) está dedicada Numini sancto deo Silvano por Succesianus Augu(sti) ser(vus); otra (CIL 11,5388 Sevilla) está dedicada por $Q$. M(arcius?) Theseus, probablemente un liberto, deo Silvano. Tampoco en ninguno de ambos casos la ubicación geográfica ni el status social de los dedicantes autorizan a pensar en un ámbito de religiosidad indígena por el mero hecho de mencionarse en ellos la palabra deus. De la misma manera sucede con un árula de Barcelona (CIL II, 4499) dedicada $d$ (is?) d(eabusque?) Silvanis por el muy romanizado en su onomástica M. Antonius Crescens. Algunos de estos casos, efectivamente, a pesar de figurar en el CIL no fueron recogidos por Lambrino; por otra parte, se habrá observado que para comprender el epígrafe como manifestación de un acto religioso es necesario tener en cuenta las dos partes que se implican en él: la divinidad a que se dedica y el dedicante

$\left.{ }^{8}\right)$ J. RODRÍGUEZ CORTÉS, Sociedad y religión clásica en la Bética romana, Salamanca 1993, pp.76-81; once epígrafes dedicados a Marte en la provincia. 
que hace la ofrenda. Hasta ahora los estudios sobre religiones indígenas sólo han tenido en cuenta los nombres de las divinidades, pero han prestado muy poca atención a los dedicantes que ofrecían las inscripciones, su estatus jurídico, su procedencia geográfica, etc.

3. El segundo supuesto de la hipótesis de Lambrino viene dado por la presunción de que es necesario restituir, al lado del denominativo deus, el nombre de una divinidad indígena. Ello sería válido si pudiéramos comprobar que en las lenguas indígenas lo habitual, si no lo obligado, era acompañar el nombre de las divinidades con algún término propio derivado del i.e. *deiwos, el término común indoeuropeo para referirse a la noción de "dios". Sin embargo, no parece que esto sea lo corriente. La consulta de las listas de teónimos realizada por Untermann en aquel anterior Coloquio muestra precisamente lo contrario; es decir, que las denominaciones compuestas por deus+teónimo son una minoría en comparación con las denominaciones en que el teónimo aparece solo o acompañado por uno o varios adjetivos, que hemos de considerar sin duda como epítetos.

El ámbito más apropiado para hacer esta comprobación parece ser sin embargo el de aquellos textos en lengua indígena que parecen contener teónimo. Podemos esperar que en ellos se nos muestre la manera propia de los indígenas de referirse a sus dioses. Para referimos a textos seguros, tomaremos solamente en consideración tres de ellos bien conocidos: la inscripción de Cabeço das Fráguas, la gran inscripción de Peñalba de Villastar y el bronce celtibérico de Botorrita.

La inscripción de Cabeço das Fráguas menciona tres teónimos sobre los que no hay dudas: Trebopala, Trebarune y Reue, y tal vez un cuarto, Laebo, antecedidos por acusativos que deben referirse a las ofrendas hechas a estas divinidades. En los cuatro casos, los teónimos aparecen solos, sin ningún denominativo del tipo $* \operatorname{deiwos}\left({ }^{9}\right)$.

$\left({ }^{9}\right)$ La inscripción, también lusitana, de Lamas de Moledo menciona una donación: doenti "dant" con su acusativo angom lamaticom y dativo crouceai macareaicoi que puede referirse al nombre de una divinidad si crouceai lo relacionamos con crougin, en una inscripción de Mosteiro da Ribeira en que aparece Crougintoudodadigoe (BRAH LVIII, 394). Tovar interpretaba el segundo elemento, teudodadigoe, como equivalente a otros en inscripciones lusitanas: una de Talaván que menciona a Munidi Eberobrigae Toudopalandiagae (BRAH,64,1914, 306), y al propio teónimo Trebopala. Todos ellos expresarían la idea de una piedra sagrada propia del pueblo, de la tribu (itrebopala) o de la nación ("teutopala). Cf. A.TovAR, "La inscripción del Cabeço das Fráguas y la lengua de los lusitanos" Actas de III Coloquio sobre lenguas y culturas paleohispánicas, 1985, pp.232-237 y nota 36 . 
La inscripción grande de Peñalba de Villastar, como es sabido, registra una dedicatoria a Lug, Lugus, mencionando en dos ocasiones el nombre del dios en dativo sing., Luguei. En ambos casos también, el nombre del dios aparece sin ningún género de denominativo.

De la misma manera, la inscripción celtibérica de Botorrita parece contener la mención segura de dos divinidades, que De Hoz restituye como Togoits y Samicios, expresadas ya desde la fórmula inicial:

Tiris[:]com.bertunetacam:Tocoitoscue:Sarnicio:cue: sua:combalces: ne litom

y que se repiten varias veces en la inscripción. En ninguno de los casos, sin embargo, parece que Togoitos y Samicios aparezcan acompañados de una palabra de signifique "dios" o "divinidad" $\left({ }^{10}\right)$.

4. En esta situación, pensamos que tal vez el análisis de los dedicantes de las inscripciones a divinidades que llevan el denominativo deus podría aclarar este problema al ser una variable que hasta ahora no se ha tenido en cuenta.

Para ello realizamos una encuesta tomando como base la lista proporcionada por Untermann a la que añadimos algunas inscripciones aparecidas posteriormente $\mathrm{u}$ otras no incluidas por él a pesar de su notoria pertinencia, como es el caso de la inscripción CIL II, 814, de La Oliva de Plasencia, dedicada Dis Laribus Gapeticorum gentilitat(is). El conjunto de la muestra nos dio un total de casi un centenar de inscripciones, 94 exactamente, en las que aparece expresa o abreviada la denominación deus, - $a$. De estas 94 inscripciones, 60 están dedicadas a la divinidad indígena Endovellicus con distintas fórmulas: deus Endovellicus, deus sanctus Endovellicus o Endovellicus simplemente. La lista hubiera sido más larga si hubiésemos incluido aquellas inscripciones en que por cualquier razón, lo más frecuentemente pérdida parcial del epígrafe, ha desaparecido el nombre de la divinidad. El conjunto de inscripciones dedicadas a Endovellicus sesga evidentemente la muestra y por ello merece un tratamiento aparte que dejaremos para otra ocasión. No obstante, queremos hacer dos observaciones por su inmediata evidencia. En primer lugar, la fórmula más habitual de mencionar este dios en las inscripciones es la de Endovellicus, con 35 casos, frente a la de deus

(10) DE HOZ, J. "La epigrafía celtibérica”, Epigrafía hispánica de época romano-republicana, Zaragoza 1986, 87. 
Endovellicus, con 19, y la de deus sanctus Endovellicus, que sólo aparece en 4 ocasiones. De acuerdo con estos datos, parece que la forma normal de mencionar la divinidad por parte de sus devotos era la de el nombre del dios solamente, como sucede en los textos e inscripciones en lengua indígena, y en este caso habríamos de ver el desarrollo de fórmulas como deus o deus sanctus como algo aportado por la romanización de estas poblaciones; es decir no como un elemento que indica la pervivencia de un culto o divinidad indígenas, sino todo lo contrario, que indica la romanización de dicho culto. En segundo lugar, la inmensa mayoría de los dedicantes presentan una onomástica latina, no indígena y solamente en un par de ocasiones podemos pensar en una onomástica mixta (.IRCP, 487: Antubellicus Priscus; 496: Helvia Avita). Todo ello lo que parece indicar es que nos hallamos en un estadio muy avanzado de romanización, cuando gran parte de la población ha perdido $o$ abandonado su onomástica original para adoptar otra de origen latino.

Desde el punto de vista social, es muy difícil clasificar este conjunto de dedicantes. En un par de ocasiones se trata de individuos pertenecientes al orden ecuestre (C/L 11,131: Sextus Cocceius Craterus Honorinus, eques Romanus, y 140: Sitnia Q.f. Victorina ex visu Q. Sitoni equestris patris); hay también varios esclavos (3-4) y podemos sospechar la existencia entre ellos también de algunos libertos (IRCP 488: Hermes [...] P.[l]ib(ertus)| 490: L.Calpurnius Andronicus?; 540: ..Aus [N]arcissus). Pero la gran masa de los dedicantes está constituida por individuos de onomástica latina, muy frecuentemente portadores de tria nomina en el caso de los hombres y duo nomina en el caso de las mujeres, que no indican sin embargo ningún otro elemento (tribu, ordo, etc.) que nos permitiera establecer su adscripción social. La solución que nos parece más aceptable es suponer que estos individuos pertenecen mayoritariamente a la plebe urbana o rústica, que debía constituir el grupo de población más numeroso en Lusitania meridional durante el Alto Imperio, entre la cual el culto de Endovellicus, con rasgos premonitorios, salvíficos y médicos, debía tener gran aceptación.

El resto de los epígrafes dedicados a divinidades indígenas acompañadas del término deus suma 36 casos. De ellos, en 22 ocasiones el dedicante lleva onomástica latina; sólo en 6 casos la onomástica del dedicante es indígena inequívocamente; y en 8 ocasiones no es posible determinar si el dedicante lleva nombre indígena o latino con seguridad, bien porque no aparezca expreso o porque haya desaparecido de la inscripción. Hemos incluido dentro de los dedicantes indígenas aquellos 
casos en que se trata de un colectivo como el ordo Zoelarum en la inscripción CIL 11,2606 (.DeoAerno ordo Zoelar(um) ex voto), o CIL 11,814 (Dis Laribus Gapeticorum gentilit(atis)). La conclusión que se desprende es que la denominación deus, - $a$ parece algo vinculado más bien a la latinización, lingüística, cultural y religiosa, que a una pervivencia del elemento indígena.

Dentro de este conjunto de inscripciones destacan por su relativa abundancia, aunque mucho menor que la de los epígrafes de Endovellicus, las inscripciones dedicadas a Dea sancta Turibrigensis Ataecina.

Será preciso recordar, a este respecto, que los dos casos concretos analizados por Lambrino como prueba de su teoría eran los de Iupiter Solutorius Eaecus y Ataecina Proserpina. En el primer caso, teníamos una serie de inscripciones, procedentes en su mayor parte del norte de Extremadura, dedicadas a Iupiter Solutorius y un epígrafe en la misma región dedicado a Deo Eaeco ${ }^{(n)}$. El nexo entre unos y otro vendría dado por un epígrafe de Poza de la Sal, que Lambrino situaba nada menos que en la Extremadura española, dedicado Iovi Solutorio Eaeco. El error geográfico explica que pasara desapercibido el hecho de que se intentaba conectar la inscripción a deo Eaeco y la serie de Iupiter Solutorius a través de un epígrafe de la provincia de Burgos, a más de $400 \mathrm{kms}$. de distancia de Coria y de Cáceres. Por otra parte, la revisión de las inscripciones de Poza de la Sal hecha por M.L. Albertos y J.A. Abásolo demostró que la inscripción de Poza de la Sal era en realidad una inscripción funeraria, dedicada a Cneo Avito Saeco, y elimina cualquier posibilidad de relacionar el culto de Eaecus con el de Iupiter Solutorius ( ${ }^{12}$ ). Algo muy importante, además, es el hecho de ninguna inscripción está dedicada a deo Iovi Solutorio, lo que hubiera sido coherente con la hipótesis de Lambrino. Ello no obstante, el culto de Júpiter Solutorio se da en un contexto claramente indígena; si tenemos

(") J.SALAS, J.A.REDONDO y J.L.SÁNCHEZ ABAL, "Un sincretismo religioso en la Península Ibérica: Júpiter Solutorio Eaeco", incluyen dos inscripciones más; una de Brozas (n. ${ }^{\circ}$ ): Cilius/Caenonis/f Apulus/Eaco/v.s.l.m., y otra de Alcántara publicada anteriormente por M. BELTRÁN (.$^{\circ} 4$ ): Balaesu/ deae/ animo/ libes volt $/$ um victoria/ solvit, en la que proponen leer: Balaesu/s C. l(ibertus) Eae[co]/ animo/ libes voltum]/ v(ovit) victoria/ solvit. A partir de la fotografía suministrada por ellos mismos, preferimos la lectura de Beltrán, ya que ni hay espacio para restituir Eae[co], ni Victoria es antropònimo frecuente en el $\boldsymbol{C I L}$ II.

(12) AbÁsolo, J.A. y Albertos, M.L. "Acerca de unas inscripciones de Poza de la Sal” BSAA XLII, 1976, pp. 393-395. 
en cuenta que la onomástica de los dedicantes es mayoritariamente indígena y que su estatuto social parece ser el de peregrinos.

En el caso de la Dea Sancta Turibrigensis Ataecina, como parece ser su titulatura completa, se hará notar que la asimilación de Ataecina a Proserpina viene dada en un único texto ( $C I L$ 11,462), una lápida de mármol que es una especie de imprecación y en la que no figura el nombre del dedicante, hallado en Mérida, donde había al parecer un culto popular a Ceres y Proserpina. En base a él Hübner restituyó las abreviaturas de CIL II, 461, una inscripción de Aldea de Cárdenas, cerca de Mérida, como D(eae) S(anctae) A(taecinae) P(roserpinae). En el resto de los epígrafes Ataecina aparece como Dea Sancta Turibrigensis, Dea Sancta, Sancta, o Dea Sancta Ataecina Turibrigensis. En la mayoría de las ocasiones los dedicantes presentan una onomástica latina (.L.Iuventius Iulianus, Nonius, PFlorius Venustus, Valerius Rufus Caepio, etc.), aparecen varios esclavos (Artemas, Claudi Martilini servus; Victorinus, servus Corneliae Severae; y Diadumenus, servus Caesaris nostri) y, solamente en una ocasión aparece una dedicante con onomástica indígena: Crastena Vitalis (filia), en una inscripción de Herguijuela, Cáceres, Sanctae sacr(um), que razonablemente podemos suponer que es una dedicatoria a Ataecina. El mismo nombre, en forma masculina, Crastuno, se documenta en San Esteban de Gormaz y Barcebalejo( $\left.{ }^{13}\right)$.

El culto de Ataecina, originariamente un culto propio de la ciudad de Turibriga o Turobriga, por alguna razón extendió su fama y su influencia fuera de los límites del territorium de esta ciudad hasta el punto de que en el Imperio parece haber sido adoptado en Augusta Emérita, de la cual o de cuyo entorno proceden varios epígrafes. Estaríamos tentados de sospechar que es esta adopción, en un marco específicamente romano, y su asociación con Proserpina lo que ha potenciado precisamente dicho culto. Es de destacar que, contrariamente a la teoría de Lambrino, CIL II no recoge ninguna inscripción a Dea Proserpina, salvo aquellos epígrafes en que Proserpina y Ataecina se asocian; y, por el contrario, las expresiones dea sancta y domina se asocian expresamente al teónimo indígena Ataecina como muestra de su compenetración con lo romano y no de ninguna supervivencia o enmascaramiento de culto indígena ya que, al mantenerse el teónimo, dicho uso sería puramente redundante.

(13) M. ${ }^{a}$ L. AlBertos, La onomástica primitiva de Hispania, Tarraconense y Bética, p. 98 
En conclusion, el estudio de los teónimos hispanos acompañados de la denominación deus,-a muestra que, contra la opinión admitida habitualmente, la utilización de dicho denominativo no indica por sí misma la pervivenda o subyacencia de un culto indígena. Su uso se da tanto con teónimos romanos como indígenas, pero sobre todo con estos últimos $\mathrm{y}$, por consiguiente, constituirían una mera redundancia si su función fuese la de enmascarar a una divinidad autóctona en trance de fusión con otra romana. Por otra parte, el uso de deus + teónimo no es extraño tampoco al latín ni a la religión romana; y, por el contrario, en los escasos textos en lenguas paleohispánicas que mencionan divinidades éstas aparecen citadas sin un término equivalente al latino dea,-us. Es este uso del teónimo solo lo que parece característico de las lenguas paleohispanas según el estado actual de nuestros conocimientos, y por el contrario la utilización de términos como deus, deus dominus o deus sanctus lo que parecen indicar es un creciente grado de romanización. 


\section{APENDICE I $\left({ }^{14}\right)$ \\ DEUS, $-A+$ TEONIMO INDIGENA}

\begin{tabular}{|c|c|c|c|c|c|c|c|}
\hline & & & & dica & & & \\
\hline & & & 'ástic & & & & \\
\hline Ref. & Teónimolat. & ind. & $e q$. & pl. & lib. & se. & per. \\
\hline CIL.II,2606 & Deo Aerno & & & $\mathrm{x}$ & & & $\mathrm{x}$ \\
\hline II,5651 & $"$ & $\mathrm{x}$ & & & & & $\mathrm{x}$ ? \\
\hline E. 83 & " & $\mathrm{x}$ & & & & & $\mathrm{x}$ ? \\
\hline II, 462 & Dea Ataecina & & & & & & \\
\hline II, 605 & " & & & & & & \\
\hline II,sup.42 & " & $\mathrm{x}$ & & & & & $\mathrm{x}$ \\
\hline II,5298 & " & $\mathrm{x}$ & & & & & $\mathrm{x}$ \\
\hline II,5999 & “" & $\mathrm{x}$ & & & $\mathrm{x}$ & & \\
\hline II,461 & " & & & & & & \\
\hline$R P H, 143$ & " & $\mathrm{x}$ & & & $\mathrm{x}$ & & \\
\hline E.116 & “ & $\mathrm{x}$ & & & $\mathrm{x}$ & & \\
\hline$A E A, 1970,144$ & " & $\mathrm{x}$ & & & $\mathrm{x}$ & & \\
\hline$A E A, 1970,146$ & " & & $x ?$ & & & & $\mathrm{x}$ ? \\
\hline$A E A, 1982,161$ & " & $\mathrm{x}$ & & & & $\mathrm{x}$ & \\
\hline$I R C P, 95$ & & $\mathrm{x}$ & & & $\mathrm{x}$ & & \\
\hline A. 57 & D.D.Segidiaeco & $\mathrm{x}$ & & & $\mathrm{x}$ & & \\
\hline II,5552 & D.D.Cusu & & & & & & \\
\hline & Neneoeco & $\mathrm{x}$ & & & $\mathrm{x}$ & & \\
\hline E,166 & D.D.Neneoeco & $\mathrm{x}$ & & & $\mathrm{x}$ & & \\
\hline II,5563 & Deo Durbedico & & $\mathrm{x}$ & & & & $\mathrm{x}$ \\
\hline A. 58 & Deo Eniragillo & & $\mathrm{x}$ & & & & $\mathrm{x}$ \\
\hline
\end{tabular}

${ }^{(14)}$ A las abreviaturas habituales de revistas y repertorios epigráficos se

añadirán las siguientes:

A = Albertos, M.L. Organizaciones s up rajami liares en la Hispania Antigua, Valladolid 1975.

$\mathrm{E}=$ Encarnação, J.d' Divindades indigenas sob o dominio romano em

Portugal, Lisboa 1975.

Ep.Astorga = Mañanes Pérez,T. Epigrafía y numismática de Astorga romana y su entorno, Salamanca 1982.

F. Fernández= Fernández Gómez,F. El santuario de Postoloboso (Candeleda, Avila), Madrid 1974.

$\mathrm{II}=\boldsymbol{C I} \boldsymbol{L}$ II

IRCP = Encarnação, J.d' Inscrições romanas do Conventus Pacensis, Coimbra 1984.

$R P H=$ BlázquezJ.M. Religiones primitivas de Hispania, Madrid 1962.

Symbolae Mitxelena = Melena, J.L. "Salama, Jálama y la epigrafía latina del antiguo corregimiento" Symbolae Ludovico Mitxelenae septuagenario oblatae, Vitoria 1985, pp. $475-530$.

Conimbriga, 34 (1995) 129-146 


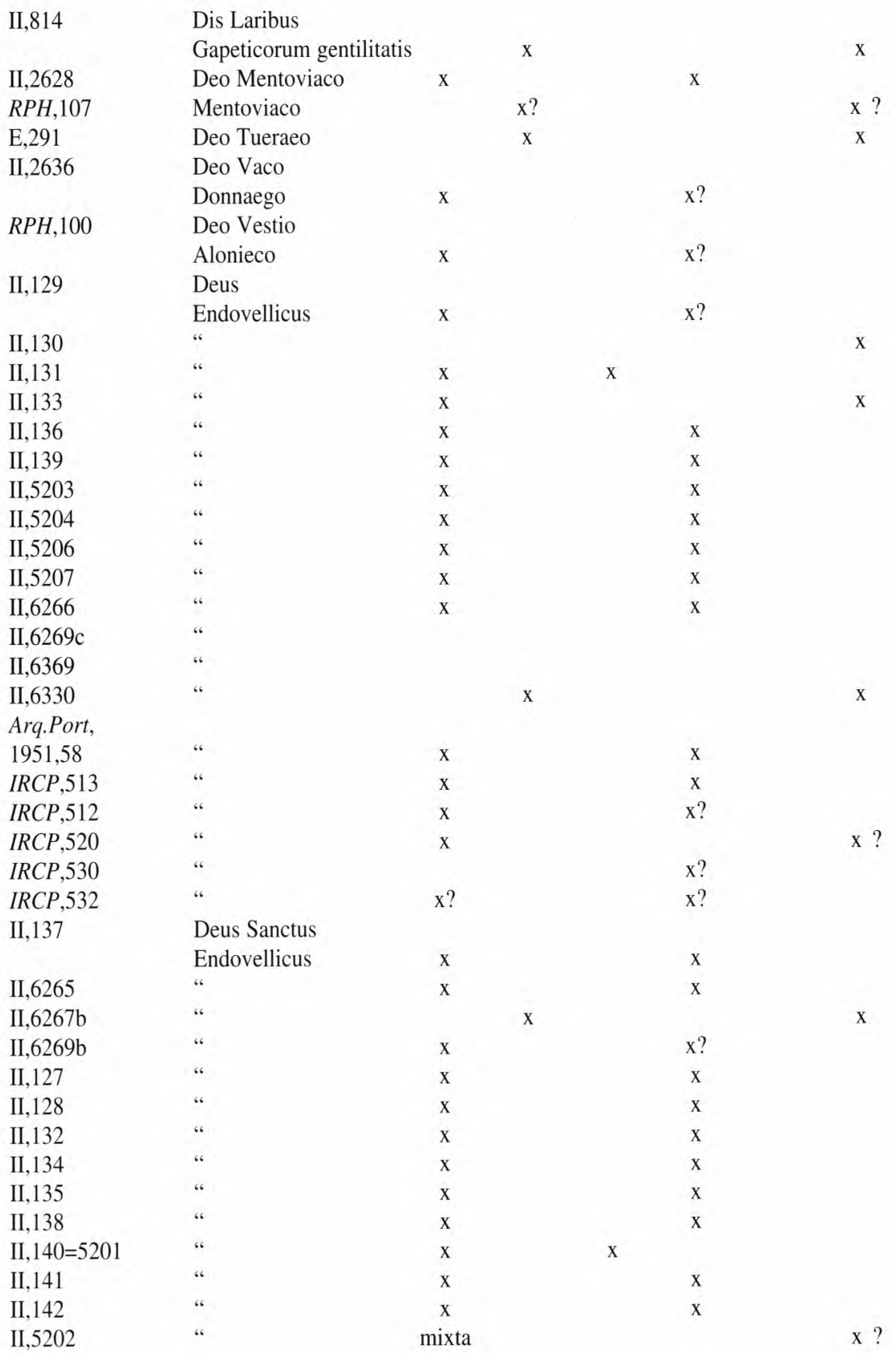

Conimbriga, 34 (1995) 129-146 


\begin{tabular}{|c|c|c|c|c|c|c|}
\hline II,5208 & " & $\mathrm{x}$ & & $\mathrm{x}$ & & \\
\hline II,5209 & “ & & & & & \\
\hline II,6265 & " & $\mathrm{x}$ & & & $\mathrm{x}$ ? & \\
\hline II,6267 & “ & mixta? & $\mathrm{x}$ & & & \\
\hline II,6267a & " & $\mathrm{x}$ & & $\mathrm{x}$ & & \\
\hline II,6268 & $"$ & $\mathrm{x}$ & & $\mathrm{x}$ & & \\
\hline II,6269 & “ & $\mathrm{x}$ & & $\mathrm{x}$ & & \\
\hline II,6269a & $"$ & $\mathrm{x}$ & & $\mathrm{x}$ ? & & \\
\hline II,6331 & " & mixta? & $\mathrm{x}$ ? & & & \\
\hline BEP,1951,101 & “ & $\mathrm{x}$ & & $x$ & & \\
\hline$B E P, 1951,101$ & “ & $\mathrm{x}$ & & $\mathrm{x}$ & & \\
\hline$B E P, 1951,25$ & “ & $\mathrm{x}$ & & $x$ & & \\
\hline$B E P, 1951,16$ & “ & $\mathrm{x}$ & & $\mathrm{x}$ & & \\
\hline$B E P, 1951,12$ & “ & $\mathrm{x}$ & & $\mathrm{x}$ & & \\
\hline$I R C P, 491$ & “ & $\mathrm{x}$ & & $\mathrm{x}$ & & \\
\hline $\operatorname{IRCP}, 501$ & $"$ & $\mathrm{x}$ & & $\mathrm{x}$ & & \\
\hline IRCP, 502 & “ & $\mathrm{x}$ & & $\mathrm{x}$ & & \\
\hline$I R C P, 505$ & " & $\mathrm{x}$ & & $\mathrm{x}$ & & \\
\hline$I R C P, 509$ & $"$ & $\mathrm{x}$ & & $x ?$ & & \\
\hline IRCP,524 & “ & $\mathrm{x}$ & & $\mathrm{x}$ & & \\
\hline $\operatorname{IRCP}, 536$ & “ & $\mathrm{x}$ & & & & $\mathrm{x}$ \\
\hline $\operatorname{IRCP}, 540$ & " & $\mathrm{x}$ & & & $\mathrm{x}$ ? & \\
\hline$I R C P, 543$ & “ & $\mathrm{x}$ & & $\mathrm{x}$ ? & & \\
\hline IRCP,498 & - & $\mathrm{x}$ & & & $\mathrm{x}$ & \\
\hline IRCP,506 & & $\mathrm{x}$ & & $\mathrm{x}$ ? & & \\
\hline \multirow[t]{2}{*}{ II,432 } & D.D. & & & & & \\
\hline & Conimbrigensium & $\mathrm{x}$ & $\mathrm{x}$ ? & & & \\
\hline II,763 & Deo Eaeco & $\mathrm{x}$ & & & & $\mathrm{x}$ ? \\
\hline \multirow[t]{2}{*}{$B R A H, 1965,140$} & Dibubus & & & & & \\
\hline & Pinionesibus & $\mathrm{x}$ & & & $\mathrm{x}$ & \\
\hline Symb.Mitxelena & Deo O. & & & & & \\
\hline 479 & Salamati & $\mathrm{x}$ & & & & $\mathrm{x}$ ? \\
\hline$B S A A, 1976,395$ & Suttunio Deo & $\mathrm{x}$ & & $\mathrm{x}$ & & \\
\hline \multirow[t]{2}{*}{ II, 2395} & Diis(numinis) & & & & & \\
\hline & Lapitearum & $\mathrm{x}$ & & $x$ ? & & \\
\hline F.Fernandez,216 & Deo Vaelico & $\mathrm{x}$ & & $\mathrm{x}$ & & \\
\hline F.Fernández,218 & Deo Velico & & & $x ?$ & & $\mathrm{x}$ ? \\
\hline F.Fernández,221 & $\mathrm{D}(\mathrm{eo}) \mathrm{V}($ aelico $)$ & - & & & & \\
\hline F.Fernández,225 & “ & - & & & & \\
\hline
\end{tabular}

Conimbriga, 34 (1995) 129-146 
APENDICE II

DEUS,-A SOLAMENTE (SIN TEONIMOS)

\begin{tabular}{|c|c|c|c|c|c|c|c|c|}
\hline \multirow[t]{3}{*}{ Ref. } & \multirow[t]{3}{*}{ Teónimo } & \multicolumn{7}{|c|}{ Dedicante } \\
\hline & & \multicolumn{3}{|c|}{ onomástica } & \multicolumn{4}{|c|}{ status } \\
\hline & & lat. & ind. & $e q$. & pl. & lib. & ser. & per. \\
\hline II,325 & Dibus & - & & & & & & \\
\hline II,5731 & “ & - & & & & & & \\
\hline II,4456 & Dibus deabusq. & $\mathrm{x}$ & & & $\mathrm{x}$ ? & & & \\
\hline \multicolumn{9}{|l|}{ II, $3025=$} \\
\hline ILER,345 & Deae D(eanae?) & $\mathrm{x}$ & & & $\mathrm{x}$ & & & \\
\hline \multicolumn{9}{|l|}{ EAE 54,1966} \\
\hline$=I L E R, 524$ & Deabus debus & & $\mathrm{x}$ ? & & & & & $\mathrm{x}$ \\
\hline Zephyrus III,54 & Dibu et debu & & $\mathrm{x}$ & & & & & $\mathrm{x}$ \\
\hline$A E A, 1970,137$ & Dis deabusq. + & $\mathrm{x}$ ? & & & $\mathrm{x}$ ? & & & \\
\hline & Mercurio et & & & & & & & \\
\hline & Manibus & & & & & & & \\
\hline \multicolumn{9}{|l|}{ Epig.Astorga, } \\
\hline \multicolumn{9}{|l|}{ Epig.Astorga, } \\
\hline $\mathrm{n}^{\circ} 11$ & $\begin{array}{l}\text { Diis deabusq. } \\
+\mathrm{IOM}+\text { Iunoni+ } \\
\text { Minervae }\end{array}$ & $\mathrm{x}$ & & $\mathrm{x}$ & & & & \\
\hline Encarnação, & & & & & & & & \\
\hline Veleia 1987,305 & Deae & $\mathrm{x}$ ? & & & $\mathrm{x}$ ? & & & \\
\hline
\end{tabular}




\section{APENDICE III \\ DEUS+TEONIMOS ROMANOS U ORIENTALES}

\begin{tabular}{|c|c|c|c|c|c|c|c|c|}
\hline \multirow[t]{3}{*}{ Ref. } & \multirow[t]{3}{*}{ Teónimo } & \multicolumn{7}{|c|}{ Dedicante } \\
\hline & & \multicolumn{3}{|c|}{ onomástica } & \multicolumn{4}{|c|}{ status } \\
\hline & & lat. & ind. & eq. & pl. & & ser. & per. \\
\hline \multicolumn{9}{|l|}{ Zephyrus } \\
\hline 1952,61 & I.M.Deo & & $\mathrm{x}$ ? & & & & & $\mathrm{x}$ ? \\
\hline ILER,5996 & Kautopates Deo & $\mathrm{x}$ & & & $\mathrm{x}$ & & & \\
\hline \multicolumn{9}{|l|}{ Zephyrus } \\
\hline 1952,59 & Deae Nem...si & $\mathrm{x}$ & & & $\mathrm{x}$ ? & & & \\
\hline
\end{tabular}

Conimbriga, 34 (1995) 129-146 